\title{
Erratum to: Sustained and differential antibody responses to virulence proteins of Brucella melitensis during acute and chronic infections in human brucellosis
}

\author{
J. Xu • Y. Qiu • M. Cui • Y. Ke • Q. Zhen • X. Yuan • \\ Y. Yu $\cdot$ X. Du $\cdot$ J. Yuan $\cdot$ H. Song $\cdot$ Z. Wang $\cdot$ G. Gao $~$ \\ S. Yu $\cdot$ Y. Wang $\cdot$ L. Huang $\cdot$ Z. Chen
}

Published online: 8 December 2012

(C) Springer-Verlag Berlin Heidelberg 2012

\section{Erratum to: Eur J Clin Microbiol Infect Dis DOI 10.1007/s10096-012-1767-7}

There was an error in the Affiliations.

The departments of the authors are modified as follows:

\footnotetext{
The online version of the original article can be found at http:// dx.doi.org/10.1007/s10096-012-1767-7.

J. Xu, Y. Qiu, M. Cui, Y. Ke, and Q. Zhen contributed equally to this work.

J. Xu $\cdot$ Q. Zhen $\cdot$ Y. Yu $\cdot$ L. Huang

Department of Epidemiology and Biostatistics,

Key Laboratory of Zoonosis, Ministry of Education,

School of Public Health, Jilin University, Changchun, Jilin

130021, People's Republic of China

Y. Qiu

Experimental Animal Center, Academy of Military Medical

Science, Beijing 100071, People's Republic of China

J. Xu $\cdot$ M. Cui $\cdot$ Y. Ke $\cdot$ X. Yuan $\cdot$ X. Du $\cdot$ J. Yuan $\cdot$ H. Song

Z. Wang $\cdot$ G. Gao $\cdot$ S. Yu $\cdot$ Y. Wang $(\bowtie) \cdot$ L. Huang $(\bowtie) \cdot$

Z. Chen $(\triangle)$

Department of Infectious Disease Control,

Beijing Institute of Disease Control and Prevention, Beijing

100071, People's Republic of China

e-mail: yufeiwang21@yahoo.com

e-mail: huangly@nic.bmi.ac.cn

e-mail: zeliangchen@yahoo.com

M. Cui

College of Veterinary Medicine,

Sichuan Agricultural University,

Ya'an 625014, People's Republic of China
} 